\title{
ECG-Based Monitoring of Electrolyte Fluctuations During the Long Interdialytic Interval
}

\author{
Ana Rodrigues ${ }^{1}$, Andrius Petrėnas ${ }^{1}$, Neda Kušleikaitė-Pere ${ }^{2}$, Pablo Laguna ${ }^{3}$, Vaidotas Marozas ${ }^{1}$ \\ ${ }^{1}$ Biomedical Engineering Institute, Kaunas University of Technology, Kaunas, Lithuania \\ ${ }^{2}$ Lithuanian University of Health Sciences, Kaunas, Lithuania \\ ${ }^{3}$ Biomedical Signal Interpretation and Computational Simulation (BSICoS) group, Aragón Institute \\ of Engineering Research (I3A), IIS Aragón, University of Zaragoza, Zaragoza, Spain and Biomedical \\ Research Networking Center (CIBER), Zaragoza, Spain
}

\begin{abstract}
Hemodialysis (HD) patients have a higher risk of sudden death due to cardiac arrhythmias, which commonly occur during the long interdialytic interval (LII) as a result of electrolyte fluctuations (EFs). Noninvasive monitoring of EFs would enable restoring normal serum electrolyte levels (SELs) by performing early HD before the onset of arrhythmias. In this study, we propose an ECGderived descriptor, $\theta_{\mu}$, that is noise robust and capable of capturing EFs during HD and the LII. To investigate the variation of $\theta_{\mu}, E C G$ and blood samples of 3 patients were acquired continuously, starting at Friday's HD and ending at Monday's HD. Results show that the increase of $\theta_{\mu}$ during Friday's HD is correlated with the decrease of SELs. Moreover, $\theta_{\mu}$ tends to decrease during the LII (no blood samples were obtained) and further increases during Monday's $H D$. If results in larger databases are confirmed, $\theta_{\mu}$ might be suitable for noninvasive monitoring of EFs during the LII.
\end{abstract}

\section{Introduction}

Life-threatening arrhythmias commonly occur in hemodialysis (HD) patients during the long interdialytic interval (LII), due to electrolyte fluctuations (EFs) [1]. Despite the compelling evidence of an increased risk of sudden death associated with abnormal serum electrolyte levels (SELs), EFs continue to be frequently undetected [2]. Thus far, EFs can only be detected through an invasive blood test, which, albeit being the only clinically accepted method of detection, is not always requested nor can be performed at home. Noninvasive remote monitoring of EFs during the LII (usually a weekend) would permit a prompt lifesaving reinstatement of normal SELs before the onset of arrhythmias.

The electrical activity of the heart is inherently depen- dent on SELs. Disturbances in the homeostatic value of SELs can, therefore, be reflected on the electrocardiogram (ECG) $[2,3]$. While electrolyte-induced changes in the ECG are well-known, several studies demonstrate a high difficulty in identifying EFs solely through an ECG $[4,5]$. This is mainly due to confounding diagnostics caused by the nonspecific nature of the emblematic ECG features. For instance, alterations in T wave and ST segment are often associated both with hyperkalemia (increased serum potassium $\left(\left[\mathrm{K}^{+}\right]\right)$levels) and myocardial ischemia [5]. The absence of identifiable ECG descriptors encumbers the ability to detect EFs accurately. Thus, exploring reliable ECG descriptors that are influenced by SELs, particularly at non-severe levels, is crucial in the development of new approaches to monitoring EFs at home. Recently, an ECG descriptor capable of determining serum $\left[\mathrm{K}^{+}\right]$levels during HD was reported in the literature [2,3]. However, these studies did not analyze the feasibility of using the descriptor to monitor EFs in ambulatory settings between HD procedures.

In this study, we propose an ECG-based descriptor capable of capturing EFs, using a wearable data acquisition equipment. To test the proposed descriptor, we studied its progression during $\mathrm{HD}$ at the time when blood was drawn and at different points in time during the LII.

\section{Methods}

\subsection{Data}

ECG and blood samples were acquired throughout the LII, starting at Friday's HD session and ending at Monday's HD. Serum potassium $\left(\left[\mathrm{K}^{+}\right]\right)$, magnesium $\left(\left[\mathrm{Mg}^{2+}\right]\right)$, calcium $([\mathrm{Ca}])$ and bicarbonate $\left(\left[\mathrm{HCO}_{3}^{-}\right]\right)$levels were evaluated. Three-lead ECGs were acquired using eMotion Faros (Mega Electronics, Finland), whereas blood was drawn for analysis at four-time points during each HD ses- 
sion: pre-HD, 30 minutes and 1 hour after the onset of HD, and post-HD. At each intended point in time, the ECGs were segmented into non-overlapping 12 minute long intervals, starting at 6 minutes before the specified time. ECGs acquired during HD were labeled at the time of each blood draw. During the LII, ECGs were analyzed at each consecutive hour commencing one hour after finishing Friday's HD.

The patient cohort comprised of 3 patients (mean age of $50.33 \pm 18.5$ years, 1 female) and each consented participating in two distinct sessions, i.e., during two LIIs. The study protocol was approved by the Kaunas Regional Biomedical Research Ethics Committee (No. BE-2-43).

\section{2. $\quad$ ECG preprocessing}

To ensure analysis of artifact-free ECGs, the segments were filtered using a zero-phase high-pass filter with cutoff frequency of $\mathrm{f}_{c}=0.5 \mathrm{~Hz}$, followed by a zero-phase $4^{\text {th }}$ order Butterworth low-pass filter ( $f_{c}=40 \mathrm{~Hz}$ ) to remove baseline wandering and noise. PQRST complexes that did not fulfill the signal quality index (SQI) criteria proposed in [6] were discarded. The valid complexes were averaged every 90 -second within the segment to further smooth the signal, ensuring a minimum of two averaged complexes per segment. The feature extraction was applied at each resultant PQRST complex.

\subsection{T wave modeling}

Abnormal electrolyte levels cause repolarization disturbances in the ventricles, which are translated into alterations in the $\mathrm{T}$ wave morphology [3]. An alternative method to quantify electrolyte-induced changes can be through the yielded parameters of model-based parameterization, a method with an increased robustness to noise that is propitious in ambulatory ECG recordings. In our previous work, we selected a lognormal function as a model for the $\mathrm{T}$ wave due to its ability to obtain an asymmetrical shape [7]. Despite showing responsiveness to morphology variations before and after HD, the model does not distinguish changes on each slope of the $\mathrm{T}$ wave separately. To address this problem, we adopted a strategy similar to the one proposed in [8] where each slope is represented by a Gaussian function. The $\mathrm{T}$ wave can then be modeled using a composite model of two distinct functions to reflect each slope individually, and is obtained as follows:

$$
T_{L G}(t)=T_{L n}(t)+T_{G}(t),
$$

where $T_{L n}(t)$ and $T_{G}(t)$ are the lognormal and Gaussian function, respectively. The right slope is defined by $T_{L n}(t)$, whereas the left by $T_{G}(t)$, as seen in Figure 1 . Each function can be characterized by its mean $(\mu)$ and standard deviation $(\sigma)$.

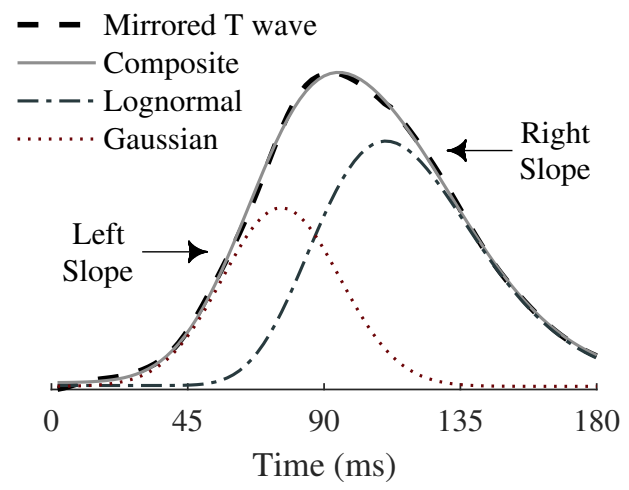

Figure 1. Illustration of the composite model fitting for a normalized $\mathrm{T}$ wave. Note that the $\mathrm{T}$ wave is mirrored.

The final parameters extracted from the composite model were, therefore, $\sigma_{R}, \mu_{R}, \sigma_{L}$ and $\mu_{L}$. The first two are derived from $T_{L n}(t)$ whereas the remaining ones from $T_{G}(t)$. Constraints were applied to the allowed ranges of all parameters to better control the fitting process. This ensures that the right slope is always fitted by $T_{L n}(t)$ and the left slope by $T_{G}(t)$.

\subsection{T wave features}

When electrolyte levels decrease during $\mathrm{HD}$, the $\mathrm{T}$ wave tends to flatten and becomes less peaked. This variation in peakedness is correlated with SELs and can be an indicator of EFs [2,3]. A possible way to measure peakedness is through $\mathrm{T}$ wave amplitude $\left(T_{A m p}\right)$, which, as several studies demonstrated, is unreliable in identifying EFs [5,7]. A hypothetical method to quantify $\mathrm{T}$ wave peakedness is through the angle, $\theta$, between the right and left slopes. Considering that each slope can be described as a line with gradient $m, \theta$ can be calculated by:

$$
\begin{aligned}
\theta & =\beta-\alpha \\
& =\arctan \left(m_{R}\right)-\arctan \left(m_{L}\right),
\end{aligned}
$$

where $\beta$ and $\alpha$ are the angles between the temporal axis and the right and the left slope, respectively, and $m_{R}$ and $m_{L}$ are the gradients of the right and left slope in $\mathrm{mV} / \mathrm{s}$, accordingly. However, when characterizing the $\mathrm{T}$ wave, variations in the duration of ventricular repolarization should also be considered. Repolarization changes are commonly reflected by a temporal displacement of each slope, which can be translated by the yielded $\mu_{R}$ and $\mu_{L}$ parameters of the composite model. As a result, each slope should be represented by both its gradient (measured from the original $\mathrm{T}$ wave after averaging) and temporal displacement (given by the composite model) as:

$$
\begin{aligned}
& S_{R}=m_{R} \cdot \mu_{R} \\
& S_{L}=m_{L} \cdot \mu_{L},
\end{aligned}
$$


where $m_{R}$ and $m_{L}$ are obtained by calculating the mean of the first derivative between T-peak and T-end point or T-beginning point, respectively. By combining (2) and (3), we then propose a new descriptor, $\theta_{\mu}$, to quantify $\mathrm{T}$ wave peakedness:

$$
\theta_{\mu}=\arctan \left(m_{R} \cdot \mu_{R}\right)-\arctan \left(m_{L} \cdot \mu_{L}\right)
$$

\section{Results}

With the restitution of normal SELs during HD, the T wave returns to its more flattened idiosyncratic morphology, which is detected by an increase of $\theta_{\mu}$, as demonstrated in Figure 2 during Friday's HD $\left(\theta_{\mu}=0.44 \mathrm{rad}\right.$ at the beginning of $\mathrm{HD}$, whereas $\theta_{\mu}=0.88 \mathrm{rad}$ at the end). During Monday's HD, the same pattern was observed between the first blood draw at the onset of $\mathrm{HD}\left(\theta_{\mu}=0.37 \mathrm{rad}\right)$ and the subsequent 30 minutes $\left(\theta_{\mu}=0.52 \mathrm{rad}\right)$. Furthermore, $\theta_{\mu}$ decreased during the LII as SELs increased and the T wave became visually more peaked.

The progression of $\mathrm{T}$ wave morphology over the course of the LII is illustrated in Figure 3. Although blood was not analyzed during the LII, we verified an increase in $\left[\mathrm{K}^{+}\right]$, $\left[\mathrm{Mg}^{2+}\right]$, and [Ca] levels on the onset of Monday's HD, whereas $\left[\mathrm{HCO}_{3}^{-}\right]$decreased, when compared to the measured levels at the end of Friday's HD. The proposed descriptor also showed a significant variation when compared to $T_{A m p}$ during the LII: changes on $T_{A m p}$ during Saturday, Sunday, and at the onset on Monday's HD are almost imperceptible, whereas changes on $\theta_{\mu}$ are more noticeable. No arrhythmias were detected during the LII in this cohort.

\section{Discussion}

This study aimed to develop an approach to capture EFs remotely before the onset of life-threatening arrhythmias in HD patients, particularly over the course of the LII, when the incidence of arrhythmias is higher [1]. Three patients were enrolled in a pilot study to evaluate the proposed descriptor and the feasibility of using a wearable device before proceeding to a larger cohort. Each patient displayed hyperkalemia, hypermagnesemia, and/or metabolic acidosis at least once on Monday which, albeit at nonsevere levels, still increases the risk of developing abnormal cardiac rhythms and supports the unmet need for noninvasive monitoring of EFs.

The progression of $\theta_{\mu}$ during HD and the LII are promising and worthy of further investigation. The proposed descriptor is responsive to variations in SELs, showing an expected increase during $\mathrm{HD}$ as the $\mathrm{T}$ wave flattened, and a decrease over the LII as the T wave slowly became more peaked. Moreover, $\theta_{\mu}$ was measured in ECG lead I solely, enabling the possibility to develop technologies that, not only monitor EFs unobtrusively but also ensure long-term wearing comfort, such as wrist-worn devices. $\theta_{\mu}$ combines the information gathered by slopes' steepnesses, which are associated with EFs during HD [2,3], with parameters derived from a parametrized $\mathrm{T}$ wave, which are advantageous in ambulatory settings due to noise robustness. Furthermore, $\theta_{\mu}$ displayed a higher sensitivity to EFs than $T_{A m p}$ and might become a more identifiable ECG descriptor of EFs.
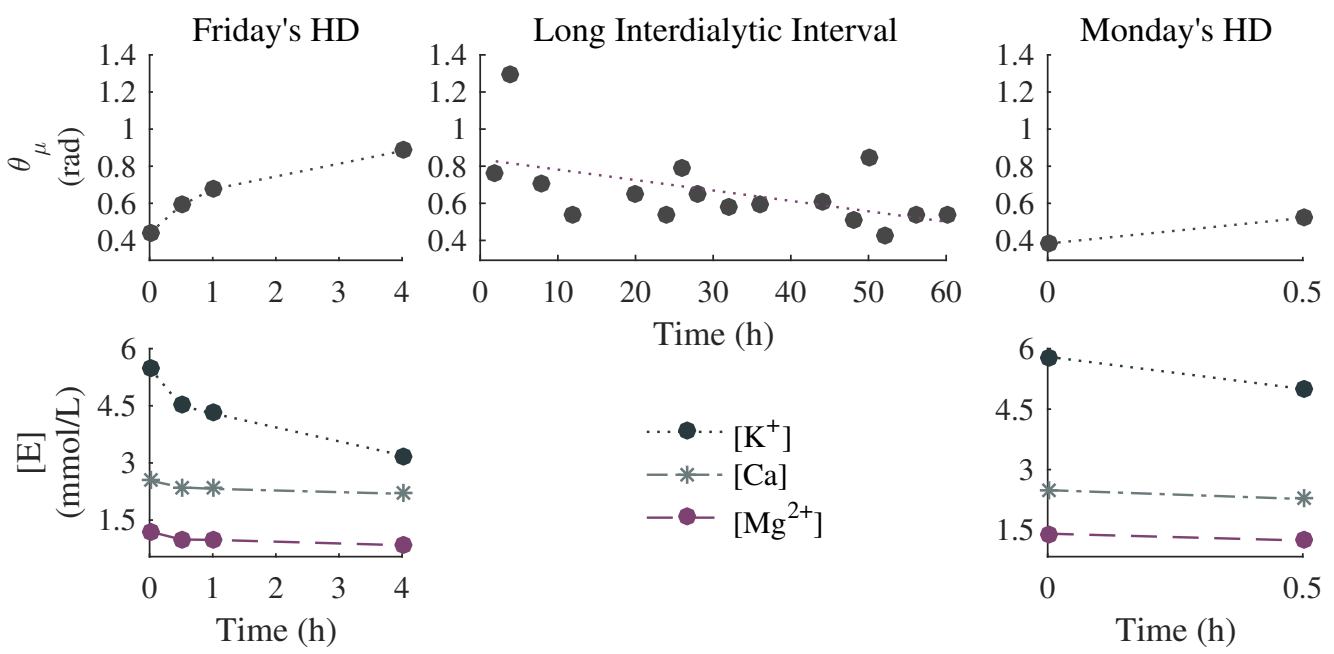

Figure 2. Variation of $\theta_{\mu}$ (top row) compared with the synchronously acquired SELs (bottom row) of one patient. $\theta_{\mu}$ was calculated at several time points after the end of Friday's HD. Although no blood samples were acquired during the LII, there was a rather expected variation of SELs, with an increment of $\left[\mathrm{K}^{+}\right]=2.6 \mathrm{mmol} / \mathrm{L},[\mathrm{Ca}]=0.3 \mathrm{mmol} / \mathrm{L}$, and $\left[\mathrm{Mg}^{2+}\right]=0.57 \mathrm{mmol} / \mathrm{L}$, and a decline of $\left[\mathrm{HCO}_{3}^{-}\right]=6.6 \mathrm{mmol} / \mathrm{L}$, between the end of Friday's HD and the onset of Monday's HD. 
a)

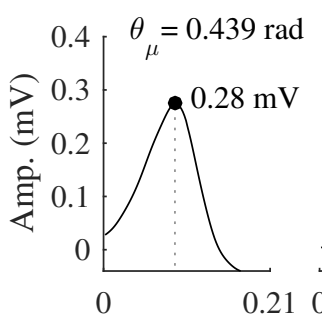

b)

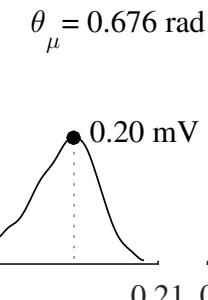

c)

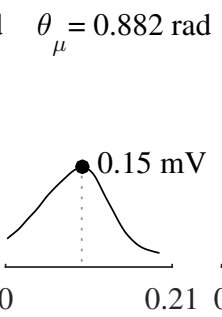

d)

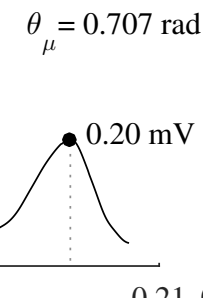

Time (s) e)

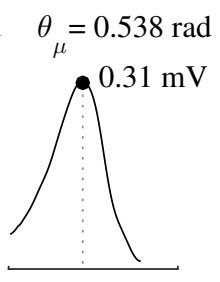

210 f)

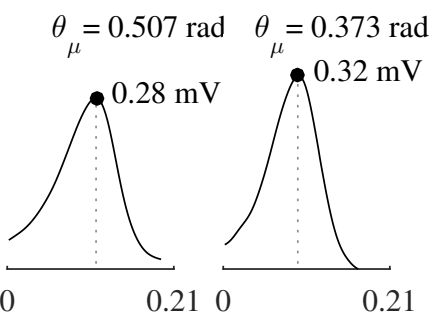

Figure 3. Illustration of the T wave morphology and the progression of $\theta_{\mu}$ at several time points: (a) onset of Friday's HD, (b) $1 \mathrm{~h}$ from the onset, (c) end of Friday's HD, (d) $4 \mathrm{~h}$ after the end of HD, (e) on Saturday after 24h, (f) on Sunday after 48h from the end of Friday's HD, (g) the beginning of Monday's HD.

Despite the encouraging initial results, this study presents several limitations, one of them being the small patient cohort. The descriptor itself also has its own limitations, mainly due to the inherent difficulties of assessing the $\mathrm{T}$ wave boundaries accurately in noisy conditions. In addition, this study did not take into consideration the influence of medications, such as potassium-lowering therapeutics and calcium supplements, on the ECG during the LII. Thus, we cannot correlate any discrepancies in the measured values of $\theta_{\mu}$ throughout the LII with such factors.

Future studies might consider exploring other descriptors in a larger data cohort and study the influence of external factors, such as medications and the circadian rhythm, on the ECG during the LII. Investigating the influence of such factors could help to reduce false alarms and develop more accurate descriptors of EFs.

\section{Conclusions}

The initial results - the variation of the proposed descriptor during HD and the LII - are encouraging and worthy of additional investigation. $\theta_{\mu}$ shows a correlation with SELs and might be suitable to be integrated into a noninvasive monitoring system of EFs during the LII between HD procedures.

\section{Acknowledgments}

This work was supported by the project "KidneyLife" funded by the EU Structural Funds under the grant No. 01.2.2-LMT-K-718-01-0030.

\section{References}

[1] Roy-Chaudhury P, Tumlin JA, Koplan BA, Costea AI, Kher V, Williamson D, Pokhariyal S, Charytan DM. Primary outcomes of the Monitoring in Dialysis Study indicate that clinically significant arrhythmias are common in hemodialysis patients and related to dialytic cycle. Kidney International 2018;93(4):941-951.

[2] Dillon JJ, Desimone CV, Sapir Y, Somers VK, Dugan JL, Bruce CJ, Ackerman MJ, Asirvatham SJ, Striemer BL, Bukartyk J, Scott CG, Bennet KE, Mikell SB, Ladewig DJ, Gilles EJ, Geva A, Sadot D, Friedman PA. Noninvasive potassium determination using a mathematically processed ECG: Proof of concept for a novel "blood-less, blood test". Journal of Electrocardiology 2015;48(1):12-18.

[3] Corsi C, Cortesi M, Callisesi G, De Bie J, Napolitano C, Santoro A, Mortara D, Severi S. Noninvasive quantification of blood potassium concentration from ECG in hemodialysis patients. Scientific Reports 2017;7:42492.

[4] Wrenn KD, Slovis CM, Slovis BS. The ability of physicians to predict hyperkalemia from the ECG. Annals of Emergency Medicine 1991;20(11):1229-1232.

[5] Montague BT, Ouellette JR, Buller GK. Retrospective review of the frequency of ECG changes in hyperkalemia. Clinical Journal of the American Society of Nephrology 2008;3(2):324-330.

[6] Orphanidou C, Bonnici T, Charlton P, Clifton D, Vallance D, Tarassenko L. Signal-quality indices for the electrocardiogram and photoplethysmogram: Derivation and applications to wireless monitoring. IEEE Journal of Biomedical and Health Informatics 2015;19(3):832-838.

[7] Rodrigues A, Marozas V, Daukantas S, Kušleikaitė-Pere N, Stramaitytė I, Bumblytė IA, Kaldoudi E. Differences between model-based electrocardiogram $\mathrm{T}$ wave features before and after haemodialysis. In 2017 IEEE Biomedical Circuits and Systems Conference (BioCAS). Oct 2017; 1-4.

[8] Clifford GD. A novel framework for signal representation and source separation: applications to filtering and segmentation of biosignals. Journal of Biological Systems 2006; 14(02):169-183.

Address for correspondence:

Ana Rodrigues

Biomedical Engineering Institute, K. Baršausko st. 59, 51423

Kaunas, Lithuania

ana.alves@ktu.lt 